\title{
Random deaths in a computational model for age-structured populations
}

\author{
J.S. Sá Martins ${ }^{\dagger}$ and S. Cebrat* \\ † Colorado Center for Chaos and Complexity, CIRES, CB 216, University of Colorado, Boulder, Colorado, USA 80309 \\ * Institute of Microbiology, University of Wroctaw, ul.S. Przybyszewskiego 63/77, 51-148 Wroctaw, Poland
}

(June 9, 2021)

\begin{abstract}
The concept of random deaths in a computational model for population dynamics is critically examined. We claim that it is just an artifact, albeit useful, of computational models to limit the size of the populations through the use of the so-called Verhulst factor and has no biological foundation. Alternative implementations of random deaths strategies are discussed and compared.
\end{abstract}

PACS numbers: 07.05.Tp, 87.23.Cc

\section{INTRODUCTION}

The concept of random deaths, an inconspicuous element of computational models designed for studies of population dynamics, has caused some controversy lately. Its main drawback comes from a strictly biological perspective: with very few exceptions, such as some human fishing practices, it is doubtful that any significant proportion of the deaths in real populations come from random causes. The usual justification for the introduction of the concept relates it to limitations on the size of the population caused by the finite amounts of food and space provided by the environment, and random deaths would be the outcome of intra-species competition for these resources. A similar argument also holds when one considers the action of predators as a limiting factor for the size of the population, and once again random deaths are summoned to account for the final effect.

In both cases, the random component of the deaths they cause is hardly significant, if at all. Genetic fitness should account for the success or failure in dealing with both constraints. The need for the concept of random deaths stems from the limited capabilities of present day models to encompass all of the relevant features of the life and death cycles of populations. It is also usually the only density-dependent regulatory mechanism, known to exist in real populations [1], provided by these models. In most cases, the concept appears under the form of the logistic, or Verhulst, factor; it has an important role in keeping the size - or, perhaps more precisely, the growth [2] - of the populations simulated in computer models within bounds through the killing of a fraction of the population due to causes not dealt with by that particular model. Its importance is thus undeniable, if one intends to study the problems of population dynamics through computer modeling. However, one must be sure that its inclusion does not affect the particular feature that the model intends to capture; otherwise, the results can be misleading. In this paper we will show that this is exactly the case for a very popular model in use today, the bit-string Penna model [3] used in the simulation of the dynamics of age-structured populations. This is done by comparing the results obtained with the use of two different versions of the implementation of the Verhulst factor.

Once the effects of the Verhulst factor are recognized, we have to choose between the two alternative implementations. The authors would be very glad not to have to deal with random deaths in their future work in the field, but could see no clear cut way of dispensing it altogether. The situation seems to be similar to the one faced by the classical statistical mechanics treatment of e.g. gases, in which events are described as random if their causes are too complicated to be analyzed precisely. We present some evolutionary arguments, together with the results of simulations of the coevolution of the two alternate populations, that favor, in the general case, one of the implementations.

\section{THE VERHULST FACTOR}

The usual implementation of random deaths in models for population dynamics is through the use of the Verhulst factor. This is a time-dependent death probability due to causes that are not dealt with by the dynamic rules of the model. Its usual form is $V(t)=N(t) /$ Popmax, where $N(t)$ is the total population at the beginning of time step $t$ and Popmax is a parameter. In the original version of the Penna bit-string model, at each time step every individual in the population, irrespective of present age or programmed death age, can be killed, with a probability $V(t)$. In what follows, we will code this strategy VA, for Verhulst equal for All. Because of the random nature of this rule, the genome space of the population is homogeneously sampled; well-fitted and ill-fitted individuals die with equal probability. As already pointed out in the Introduction, we can see no biological justification for this randomness. In a competition for limited resources and in the struggle to escape predation, better fitted organisms would most 
certainly be killed with a smaller probability. From another point of view, the economics of such random deaths would certainly be too costly, since it would expose equally to a premature killing individuals for which different investments had been made. Since the only valid reason for the Verhulst factor to still be part of the overall dynamic rules lies in the need to keep the size of the population limited, it would be in principle desirable to find an alternative and less costly strategy population-wise for its implementation.

Such an alternative has recently been suggested [4]. Instead of acting as a random death probability for all the population, the Verhulst factor acts only on the individuals whose genomes have not been tested by the environment yet, the newborn (VB). In fact, a similar strategy for the Verhulst factor was adopted by a recent simulation of the Penna model on a lattice [5]. From the economics of the population, this is clearly a better choice, since little investment is wasted. From the biological perspective, although it is not yet the most faithful representation of the real natural processes, it has an advantage since the genome of the newborn is on the average less well-fitted, because of the overwhelming majority of bad over good mutations. Random deaths will only occur for a fraction of the population that has more bad mutations than the average, and we claim that this strategy brings the model closer to reality. It is in fact known that density-dependent components of the demographic parameters respond to and affect usually only the numbers of individuals in a restricted sub-group of the population, called the critical age-group [1].

A word of caution is in order here. When simulating the evolution of VB populations, one must take special care with the initial transient, which can generate populations larger than Popmax.

\section{THE PENNA MODEL}

We will briefly describe in this section the main features of the Penna model used in our simulations. For a detailed description of the model, together with a complete set of references for work already published on it, we direct the reader to Ref. [6].

The genome of each organism is represented by two computer words. In each word, a bit set to one at a locus corresponds to a deleterious mutation - a "perfect" strand would be composed solely of zeros. The effect of this mutation may be felt by the individual at all ages equal to or above the numerical order of that locus in the word. As an example, a bit set to one at the second position of one of the bit-strings means that a harmful effect may become present in the life history of the organism to which it corresponds after it has lived for two time periods. The diploid character of the genome is related to the effectiveness of the mutations. A mutation in a position of one of the strands is felt as harmful either because of homozygose or because of dominance. For the former, a mutation must be present in both strings at the same position to be effective. The concept of dominance on the other hand relates to loci in the genome in which a mutation in just one strand is enough to make it affect the organism's life. The life span of an individual is controlled by the amount of effective mutations active at any instant in time. This number must be smaller than a specified threshold to keep the individual alive; it dies as soon as this limit is reached.

Reproduction is modeled by the introduction of new genomes in the population. Each female becomes reproductive after having reached a minimum age, after which it generates a fixed number of offspring at the completion of each period of life. The meiotic cycle is represented by the generation of a single-stranded cell out of the diploid genome. To do so, each string of the parent genome is cut at a randomly selected position, the same for both strings, and the left part of one is combined with the right part of the other, thus generating two new combinations of the original genes. The selection of one of these complete the formation of the haploid gamete coming from the mother. For

mating, a male is randomly selected in the population and undergoes the same meiotic cycle, generating a second haploid gamete out of his genome. The two gametes, one from each parent, are now combined to form the genome of the offspring. Each of its strands was formed out of a different set of genes. The next stage of the reproduction process is the introduction of $M$ independent mutations in the newly generated genetic strands. In this kind of model it is normal to consider only the possibility of harmful mutations, because of their overwhelming majority in nature. The gender of the newborn is then randomly selected, with equal probability for each sex.

The passage of time is represented by the reading of a new locus in the genome of each individual in the population, and the increase of its age by one. After having accounted for the selection pressure of a limiting number of effective harmful mutations and the random action of the Verhulst dagger, females that have reached the minimum age for reproduction generate a number of offspring. The simulation runs for a pre-specified number of time steps, at the end of which averages are taken over the population. Typically, measures are taken for the age structure of the population - number of individuals and probability of survival and death by genetic causes for each age group - as well as for the genetic composition distribution. 


\section{SIMULATION RESULTS}

Our claims are supported by the results of simulations performed with the bit-string Penna model in which we compare the outcome produced by each of the strategies outlined in the last section. First we show that the genetic patterns produced by the alternate strategies are not the same. Figure 1 shows the age distribution generated by both strategies. A number of striking differences, apart from the overall concavity of the curve, should be noted:

- The maximum life span is considerably larger for the VB population.

- The average age of an individual is larger for the VB population.

- The fraction of the population with reproductive life (age $>10$ ) is also larger for the VB population. As a consequence, the number of offspring generated at each time step and the population at equilibrium for the same value of the parameter Popmax are also larger.

A second comparison is shown in Figure 2. The pattern of fixation of alleles in the genome configuration is shifted upwards in the VB population, shrinking the size of the irrelevant (non-selective) part of the genome and corresponding to the larger life span shown in the previous plot. Figure 3 shows yet another feature that is sensitive to the choice of random deaths strategy. Here the fraction of defective genes in the population is computed for each locus. These last two plots show that the genetic configurations of both populations look pretty much the same before the age of reproduction. This is not surprising since, for a threshold of deleterious mutations of 1 , for a genome to be able to spread throughout the population it has to keep the fraction of defective genes and of homozygotes at the same low level before the onset of the reproductive period, for both populations. During this pre-reproductive period, the random deaths are responsible for the faster decrease of the age distribution of the VA population (Fig. 1). On the other hand, the genomes show a clear distinction in the reproductive period, with a slower rate of degradation for the VB population. The better quality of the genomes of the VB population is proven by the smaller fraction of deleterious mutations that they carry at each age in this period.

The results of our simulation are in fact somewhat surprising. One would naively expect, for instance, the fraction of random deaths to be larger in a VA population, since in this case the Verhulst dagger is allowed to act during the entire life span of each individual. Table shows that this is not the case. In fact, simulations show that the fraction of random deaths in the VB population is more than twice that for the VA population. The probability of random death over the whole life span is also larger for the VB population, in spite of only acting on the newborn; for this population, this probability is also the fraction of random deaths for the newborn.

A "coup de grace" in the set of comparisons we are reporting is the outcome of a simulated coevolution of the two populations. We call the reader's attention again to numerical problems that may easily be - and have already been, at least by one of us! [7] - overlooked. For any of the strategies discussed here, the role of the parameter Popmax has to be conveniently downsized. It is very often considered directly in the biological sense as a real measure of the capacity of the environment. But we argue here that this cannot be so. In fact, in simulations of the case of the genes chronologically switched on, with the Verhulst factor killing at each time step, the size of a population living in a given environment depends on the length of the genome, which has no biological justification. There seems to be no biological justification either for a VB population to be of a much higher size than a VA population, if both are simulated with the same value for Popmax, or for an asexual variety to grow larger than a sexual one of the same species. In a simulation of the coevolution of VA and VB populations without any correction of the Popmax parameter, the VA population gets extinct in the first tenths of time steps of coevolution. We claim that this is merely an artifact of the model, and that the effective carrying capacity of an environment has to be related to the value of the population at equilibrium, and cannot be different for different strategies of implementation of the concept of random deaths. If one tries to carry a realistic coevolution simulation, the parameter Popmax has to be adequately manipulated in order to make sure that the size of each population is approximately the same at equilibrium, when evolving separately.

Another important point concerns the stage, during one time step of the evolution, at which one has to probe the size of the population for that purpose. To understand this point, one has to look back at how these simulations are performed. There are some alternatives, but roughly one proceeds at each time step sequentially through the population of males and females determining the ones that are killed by genetic causes or by random deaths, and then mate them to generate the newborn. Since we proceed in a sequential manner, at each time step the population oscillates between a minimum value, which is its value after all deaths for that particular time step have been considered, and a maximum one at the end of the time step, after all births. These cycles of compression (deaths) and expansion (births) are not real, but merely artifacts of a sequential processing. It is usual to compute the total population at the beginning of a time step. This means that it includes all the individuals of $a g e>0$ that remained alive after the completion of the last time step plus the newborn. However, as above explained, this is a peak value 
for a fluctuating population, and can never actually be seen, for the dying and breeding processes happen in parallel in reality. The actual population for the purposes of comparison with the real carrying capacity of an environment cannot be taken at this peak value, but rather at some smaller one. Since the pressure of the newborn over the capacity of the environment can be neglected in the presence of that of the individuals with age $>0$, we chose to pick the minimum value of the population at each time step as the real one.

Once these considerations are taken into account, the parameter Popmax has to be compressed by some factor for the VB population to ensure that its size at equilibrium matches that of the VA population, all the other parameters being equal. Figure 4 shows the result of simulating the coevolution of the two populations. After having evolved independently for some time, to wash out any transient behaviour, the populations are brought into contact, sharing the same environment. The VA population dies out after less than 5,000 time steps. This outcome is a convincing support for the claims previously made. Nature would, if necessary, rather sacrifice those upon whom less investment has been made, as it often does in animal populations.

We address finally the question of diversity. Would a VB strategy decrease the genetic diversity provided by sexual reproduction? One might think so, since random deaths are concentrated on a fraction of the population with a larger number of mutations. Figure 5 supports a negative answer. Diversity is measured through a histogram of Hamming distances across the population, defined as the number of different alleles (bits) in every pair of genomes [8]. The VB strategy even gives diversity a small enhancement.

\section{CONCLUSIONS}

In Nature, at least in the case of higher diploid organisms, whose populations are simulated by the Penna model, random deaths play no significant role, and computational models that want to capture the essence of evolution must take this into account. We have shown that the choice of strategy in implementing this concept, which is unfortunately necessary to prevent unlimited growth of the population in those models, has an unexpected impact on its genetic profile. We claim that the choice of exposing only the newborn to random deaths is at present the most realistic one, and limits the aforementioned impact to a minimum. As an illustration, we presented an evolutionary argument based on the simulated competition of two non-crossing varieties of the same species where the outcome shows that Nature would most probably choose the same strategy as we did. It is our impression that some of the results obtained with the use of the models that rely on random deaths for their stabilization should be revised in light of the present discussion.

\section{ACKNOWLEDGMENTS}

D. Stauffer was responsible for arranging our meeting in the virtual space; we want to thank him for that and for his encouragement and intellectual support. J.S.S.M. was supported by DOE grant DE-FG03-95ER14499 and C.S. by UW grant $2027 / \mathrm{W} / \mathrm{Imi} / 2000$.

[1] B. Charlesworth, Evolution in age-structured populations, 2nd edition, Cambridge University Press (1994).

[2] A. Raab, J. Stat. Phys. 91, 1055 (1998).

[3] T.J.P. Penna, J. Stat. Phys. 78, 1629 (1995).

[4] E. Niewczas, S. Cebrat and D. Stauffer, Theory Biosci. 119, 122 (2000).

[5] D. Makowiec, Acta Phys. Polon. B 31, 1037 (2000).

[6] S. Moss de Oliveira, P.M.C. de Oliveira and D. Stauffer, Evolution, Money, War and Computers, Teubner (1999).

[7] J.S. Sá Martins, Phys. Rev. E 61, R2212 (2000).

[8] J.S. Sá Martins and S. Moss de Oliveira, Int. J. Mod. Phys. C 9, 421 (1998). 


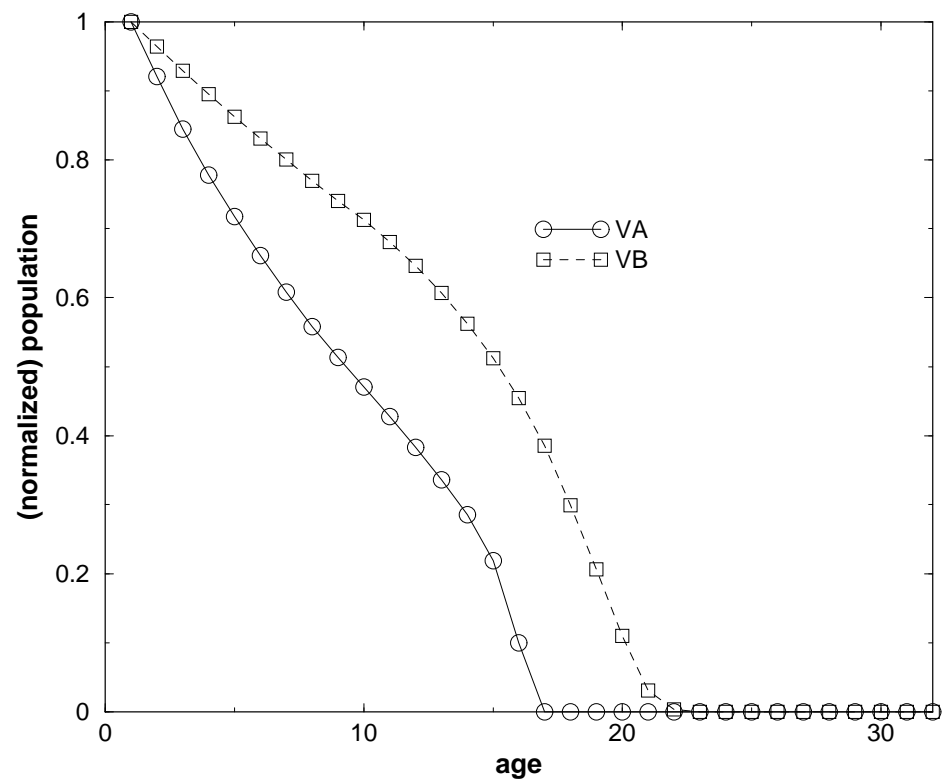

FIG. 1. Age distribution generated by random deaths over the entire population (VA) and only over the newborn (VB). The plot shows the population with a certain age, normalized to the population with age $1(N($ age $) / N(1))$. Unless otherwise stated, this and the following plots come from simulations of iteroparous populations where the genomes were 32-bits long, the minimum age at reproduction was 10 , and the threshold for deleterious mutations 1 .

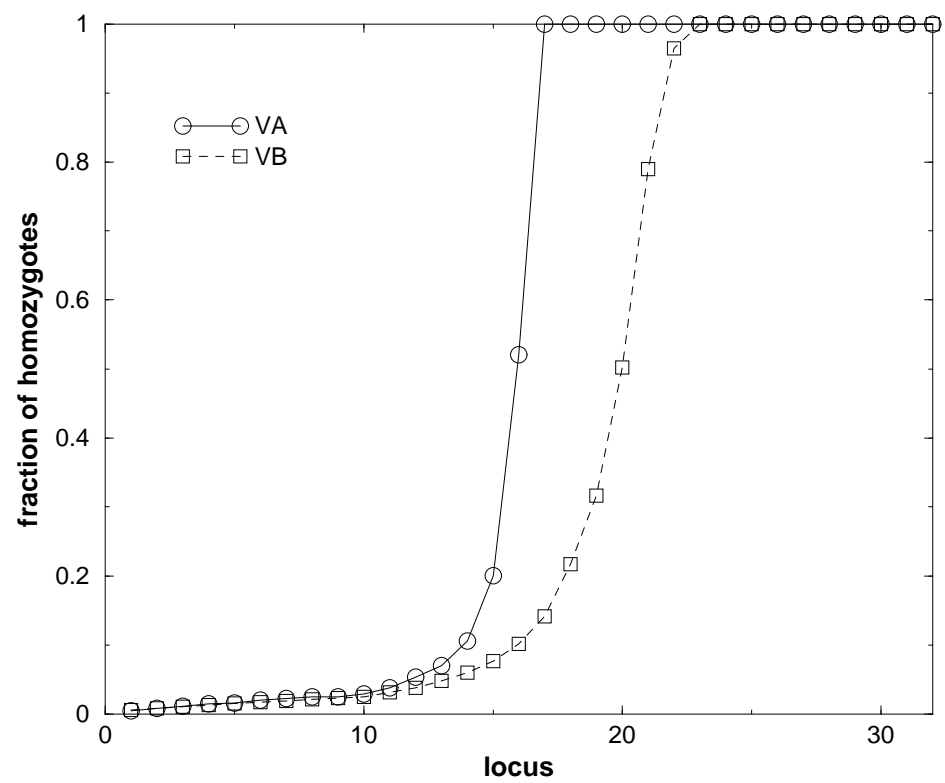

FIG. 2. The fraction of homozygotes (both bit-strings with a bit set at the location) is plotted for each locus in the genome. The simulations were run with no dominance, i.e., for a genome to express a defective phenotype, the individual had to be an homozygote at a locus. 


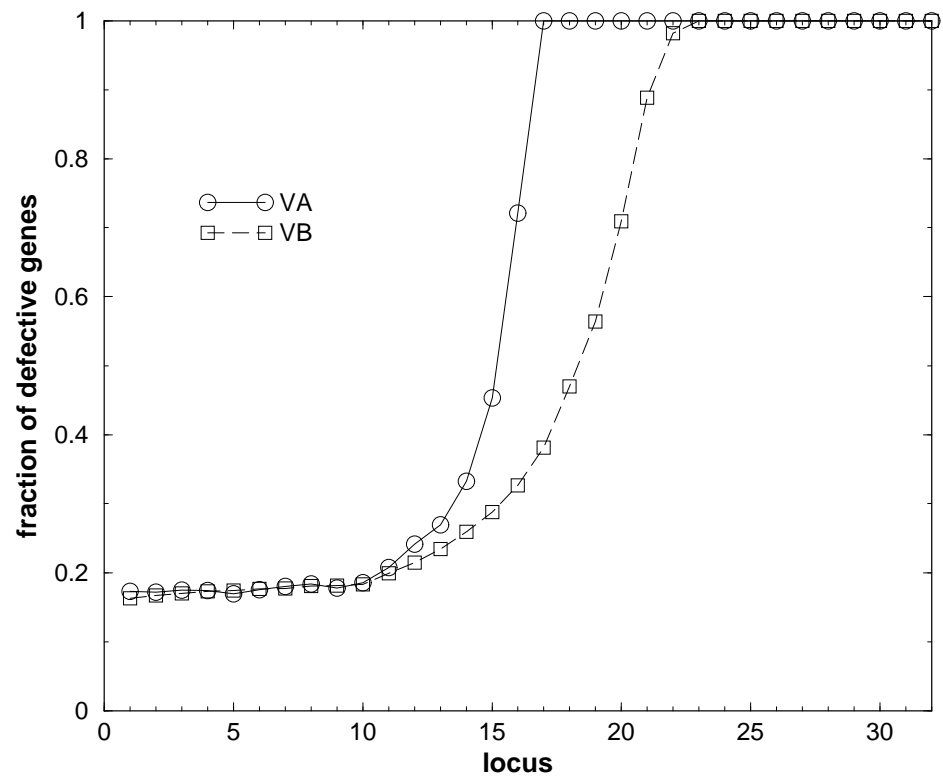

FIG. 3. The fraction of defective genes (alleles set to one at a location) is plotted for each locus in the genome.

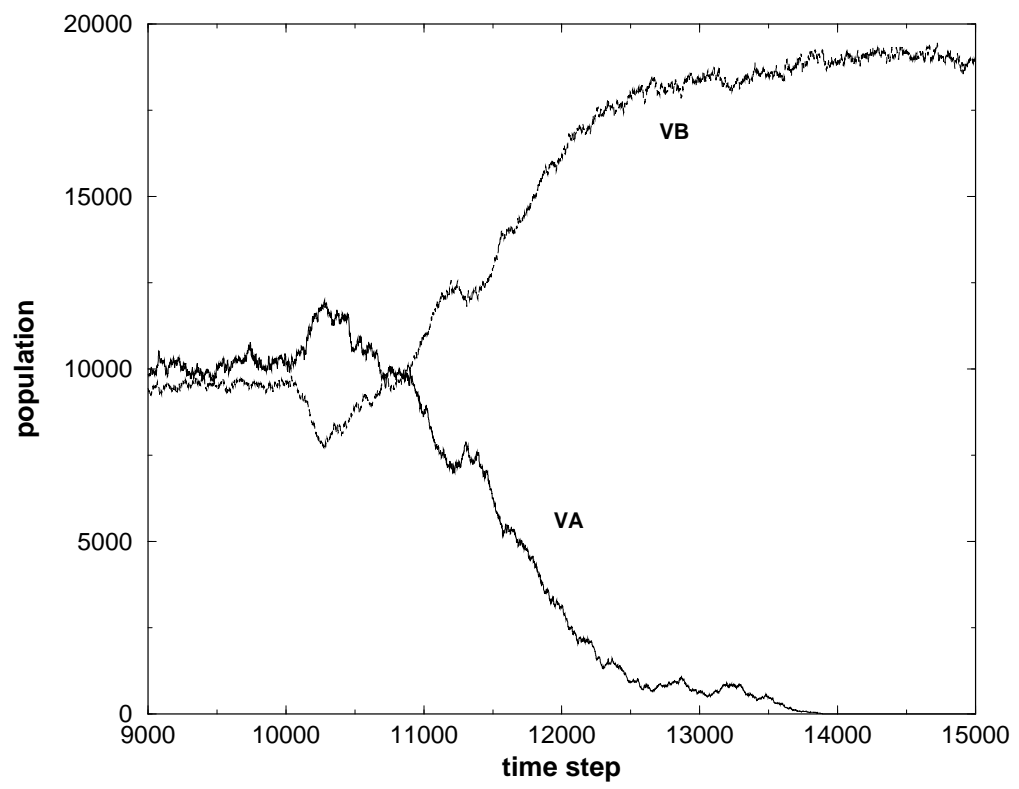

FIG. 4. The population for each variety is plotted against time. Coevolution starts at time step 10,000, after a sufficiently long transient. For the parameters we are using, in particular Popmax $=200,000$, the compression factor to be applied for the VB population to ensure equal populations of $\approx 10,000$ in equilibrium is 10.5 , leading to an effective value of Popmax $\approx 19,000$ for this population; in fact, this factor is a little bit too large, as can be seen in the plot through a slightly larger VA population at the beginning. In less than 5,000 steps the VA population dies out. 


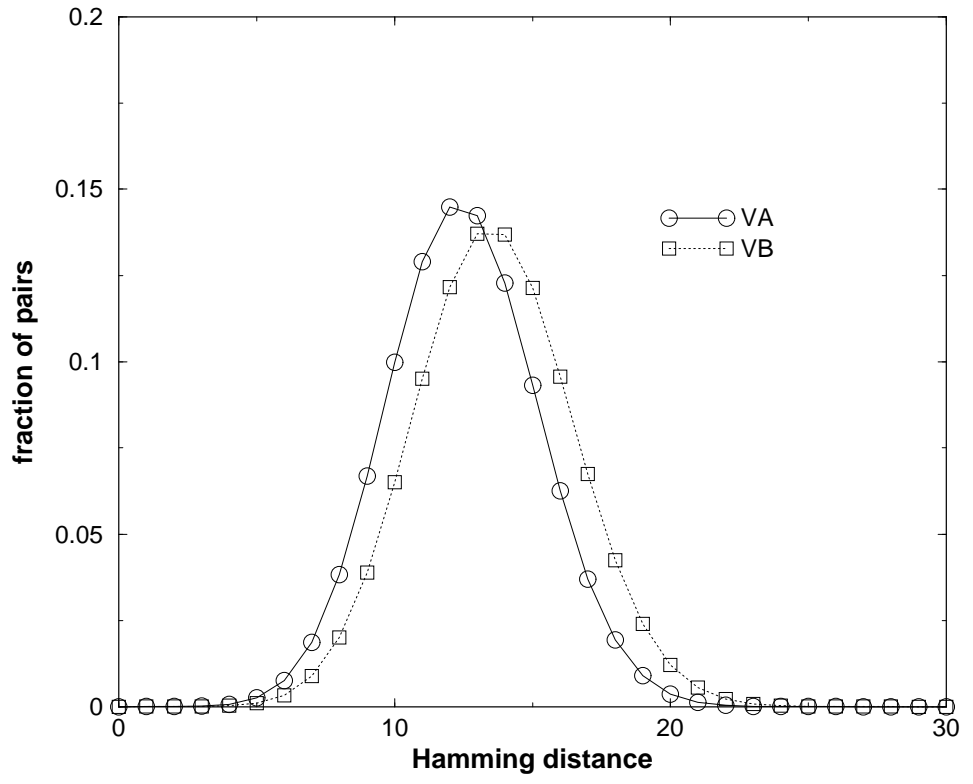

FIG. 5. Histogram of Hamming distances for both populations. The plot shows the fraction of all pairings that share each value for the distance.

TABLE I. Births and random deaths for both strategies. In the last row, we show the probability of dying by the Verhulst dagger over the entire life span.

\begin{tabular}{l|r|r}
\hline \hline & VA & VB \\
\hline births/population & 0.111 & 0.167 \\
random deaths/population & 0.043 & 0.097 \\
random deaths/births & 0.387 & 0.580 \\
probability of random death & 0.443 & 0.580 \\
\hline & & \\
\hline \hline
\end{tabular}

